\title{
Being safe, feeling safe, and stigmatizing attitude among primary health care staff in providing multidrug-resistant tuberculosis care in Bantul District, Yogyakarta Province, Indonesia
}

\author{
Ari Probandari ${ }^{1,2^{*}}\left(\mathbb{D}\right.$, Hary Sanjoto ${ }^{2}$, Melani Ratih Mahanani ${ }^{2}$, Luthfi Azizatunnisa ${ }^{2}$ and Sampir Widayati ${ }^{3}$
}

\begin{abstract}
Introduction: Patient-centered care approach in multidrug-resistant tuberculosis care requires health worker safety that covers both being safe and feeling safe to conduct the services. Stigma has been argued as a barrier to patient-centered care. However, there has been relatively little research addressing the issues of safety and stigma among health staff. This paper explored the issue of being safe, feeling safe, and stigmatizing attitude among health staff working with multidrug-resistant tuberculosis cases in primary health care facilities in Indonesia.

Methods: Using a mixed methods research design, data was collected with structured questionnaires among 123 staff, observations of infection control in 17 primary health care facilities, and in-depth interviews among 22 staff.

Results: The findings showed suboptimal infection control infrastructures for the primary health care facilities. The knowledge and motivation to follow multidrug-resistant tuberculosis care protocols are suboptimal. Feeling unsafe is related to stigmatizing attitude in providing multidrug-resistant tuberculosis care.
\end{abstract}

Conclusion: Being safe, feeling unsafe, and stigmatizing attitude are challenges in providing patient-centered multidrug-resistant tuberculosis care in primary health care facilities in Indonesia. Serious efforts are needed on all levels to ensure safety and prevent irrational stigma.

Keywords: Safety, Stigma, Health workers, Tuberculosis, Multidrug-resistant tuberculosis

\section{Introduction}

A patient-centered care approach has been considered as an essential pillar for tackling tuberculosis (TB) global epidemic [1]. Improving a patient-centered care approach, however, cannot be implemented effectively without simultaneously managing the safety of health workers, and it has been recognized that the safety of health workers is an imperative that should receive more serious attention [2].

\footnotetext{
* Correspondence: ari.probandari@staff.uns.ac.id

'Department of Public Health, Faculty of Medicine, Universitas Sebelas Maret, Jalan Ir. Sutami 36A, Surakarta 57126, Indonesia

${ }^{2}$ Disease Control Research Group, Faculty of Medicine, Universitas Sebelas

Maret, Jalan Ir. Sutami 36A, Surakarta 57126, Indonesia

Full list of author information is available at the end of the article
}

Health worker safety, however, is not just a matter of being safe. Health workers must also feel safe. In the absence of this broader health worker safety, there is a danger that patient-centered care will be jeopardized because of stigmatizing attitudes toward TB patients by unsafe health workers. Indeed, there is a significant body of literature looking at stigmatizing attitudes among health staff working in TB care, and the adverse consequences on care [3-5].

Indonesia is one of 27 countries with a high burden of multidrug-resistant tuberculosis (MDR-TB) globally [6]. Notwithstanding the burden of MDR-TB in Indonesia and the known impact of stigma on the provision of patient-centered care, there has been a limited number of research addressing the issue. This is particularly

(c) The Author(s). 2019 Open Access This article is distributed under the terms of the Creative Commons Attribution 4.0 International License (http://creativecommons.org/licenses/by/4.0/), which permits unrestricted use, distribution, and 
unfortunate because it is well known that levels of stigma, as a social construct, vary with context $[5,7]$.

The Indonesian Ministry of Health has promoted direct observed treatment (DOT) $[8,9]$ as the strategy in the management of TB. Since 2014, there has been a concerted effort to identify the primary health care facility (PHC) closest to MDR-TB patient's address as the center of care. A primary health care facility should provide treatment management for MDR-TB patients at the continuation phase.

Bantul district, a district in Yogyakarta Province of Indonesia, adopted the policy of DOT for MDR-TB at PHC settings in 2014. However, in 2016, there was the rejection of staff at PHCs to conduct DOT service for MDR-TB. This paper explored the issue of safety, feeling safe, and stigmatizing attitude among health staff working with MDR-TB cases in PHCs in Bantul district, Yogyakarta Province, Indonesia.

\section{Methods \\ Research settings}

Bantul district is one of five districts in Yogyakarta Province of Indonesia, with 971,000 inhabitants. The population density is nearly 2000 per square kilometer. There are 27 PHCs located in 17 sub-districts of Bantul [10]. Seven of 27 PHCs have implemented DOT. The number of presumptive MDR-TB cases has increased in the last 3 years, i.e., 31 cases in 2013, 41 cases in 2014, and 45 cases in 2015 (District Health Office of Bantul, unpublished data).

\section{Study design}

We applied a concurrent nested mixed methods design [11] that combined a health care facility-based cross-sectional study and a qualitative study. The cross-sectional study was conducted to assess the health staff's knowledge about infection control and their attitude to conduct proper care protocols for MDR-TB. Through the survey, we would like to describe whether health staff's knowledge about infection control and the attitude to conduct DOT for MDR-TB were lacking. The cross-sectional study is nested in the qualitative observations of infection control infrastructure and staff behavior. The observation aimed to assess the infrastructure and activities related to infection control. We also conducted in-depth interviews to explain the findings from the cross-sectional study and observation, and to explore about reasons behind irrational stigma behavior.

\section{Research participants and sampling}

For the survey, we selected staff that met the inclusion criteria in all 27 PHCs: health staff involved in implementing protocols for MDR-TB care (registration staff, medical doctors, nurses, lab staff) and infection control (staff responsible for health promotion and environmental health). Sample size calculation was done based on the formula for a descriptive cross-sectional study [12]. By estimating that 50\% of staff among total 180 population of relevant health staff at PHCs (Bantul district health office 2015, unpublished data) were knowledgeable about the MDR-TB care procedure and infection control, $95 \%$ confidence level, and precision $10 \%$, we calculated a minimum sample size of 123 individuals for our study. Data on eligible staff were obtained from the District Health Office. We selected the staff by quota sampling until we reached the minimum sample size.

For the in-depth interviews, we selected health staff at PHCs relevant to MDR-TB care by purposive sampling (medical doctor, nurses, lab staff, and TB program staff) for in-depth interviews. We also conducted observations of infection control facilities with a TB infection control consultant to assess the adequacy of infrastructure and procedures of MDR-TB care in PHCs. Both in-depth interviews and observations were conducted until data saturation was achieved.

\section{Data collection}

Two trained surveyors collected data from surveys among PHC staff under the supervision of the last author. The first and second authors mainly conducted both in-depth interviews and observations of staff behavior in conducting DOT for MDR-TB patients. An infection control expert conducted the observation of infection control infrastructure and proper MDR-TB protocols.

Structured questionnaires about knowledge on infection control and observation guidelines were developed based on the national guideline for infection control related to TB control programs at the health care facility level [13]. The questionnaires and the observation guidelines were piloted and validated. The content validity of the quantitative questionnaires and guideline of in-depth interviews were assessed through the panel of experts during the proposal finalization workshop. We piloted the quantitative questionnaires to 15 primary health care staff to assess their clarity to each question. The observation checklist of infection control was validated by the expert from Universitas Gadjah Mada, Indonesia.

\section{Data analysis}

Qualitative data were analyzed using the content analysis technique $[14,15]$. From the text of transcripts, we identified phrases/sentences containing specific meanings (meaning units). The manifest meanings and latent meanings were generated from the meaning units, which were assigned as codes. The codes with similarity were grouped into categories. Open Code software [16] was used for facilitating the data analysis. To improve the trustworthiness of the qualitative data in the data analysis, we conducted peer-debriefing in the researcher team. 
Quantitative data were used for triangulation of the qualitative data in the mixed methods design. Quantitative data were analyzed by comparing the knowledge and attitude of staff by their characteristics.

\section{Research ethics}

Ethical approval was obtained from the Ethics Committee, Faculty of Medicine, Universitas Sebelas Maret at Surakarta Indonesia. We obtained written informed consent from all study participants. The researchers conducted the informed consent for the qualitative data collection, while two enumerators conducted the informed consent for the quantitative data collection. The enumerator received training from co-PIs to conduct the informed consent process and collecting data.

\section{Results}

\section{Characteristics of study participants}

A total of 22 in-depth interviews were conducted in 17 PHCs, with 19 in-depth interviews with clinical staff (7 physicians, 1 dentist, 9 nurses, and 2 lab staff) in 17 PHCs, and key informant interviews among three TB program managers at the district/provincial level. Ten out of 19 clinical staff were also acted as TB program staff.

A total of 123 health staff in all 27 PHCs were included in the cross-sectional study (Table 1). The majority of the study informants were clinical staff (medical doctors, nurses, lab staff). On average, the study informants were in the productive age $(39 \pm 12$ years old).
Most of them were female (68\%), with the level of education of 3 years Diploma. About $90 \%$ of health staff has been working for more than 5 years.

\section{Safety}

The observation in 17 PHCs showed suboptimal infrastructure and fidelity of activities to infection control protocols as per the national guideline (Table 2). Proper layout and zoning were only available in eight out of the 17 PHCs; unfortunately, only one PHC provided DOT for MDR-TB had optimal zoning. In addition to zoning, the suboptimal infrastructure of infection control was shown for other types of infrastructures including exhaust fan, infectious disposal system, and sputum collection area. On the other hand, the availability of infectious waste disposal and surgical masks for patients with a cough were adequate.

The observations also revealed unsafe practices such as health staff's unawareness about the direction of airflow while they were providing DOT. The health staff even stood in the opposite direction of airflow. The observation also found the health staff unknowingly allowed the MDR-TB patients to be without surgery masks when the patients were having cough during the DOT.

The cross-sectional study revealed that the knowledge of health staff about infection control protocols was inadequate in some domains (Table 3). Health staff had relatively sufficient knowledge about the disposal of tissues used to close mouth when coughing (84\%).

Table 1 Characteristics of primary health care staff participating for the cross-sectional study

\begin{tabular}{|c|c|c|c|c|}
\hline Characteristics & All $(N=123)$ & Clinical staffa $(N=95)$ & $\begin{array}{l}\text { Tuberculosis program } \\
\text { staff }(N=10)\end{array}$ & $\begin{array}{l}\text { Other }^{\mathrm{b}} \text { staff } \\
(N=18)\end{array}$ \\
\hline Age (years), mean \pm SD & $39 \pm 12$ & $38 \pm 12$ & $35 \pm 14$ & $47 \pm 8$ \\
\hline \multicolumn{5}{|l|}{ Gender, $n(\%)$} \\
\hline Male & $39(31.7)$ & $27(28.4)$ & $0(0)$ & $12(66.7)$ \\
\hline Female & $84(68.3)$ & $68(71.6)$ & $10(100)$ & $6(33.3)$ \\
\hline \multicolumn{5}{|l|}{ Level of education, $n$ (\%) } \\
\hline Diploma & $68(55.3)$ & $48(50.5)$ & $9(90.0)$ & $11(61.1)$ \\
\hline Undergraduate & $46(37.4)$ & $39(41.1)$ & $1(10.0)$ & $6(33.3)$ \\
\hline Graduate & $2(1.6)$ & $1(1.1)$ & $0(0)$ & $1(5.6)$ \\
\hline Professional & $7(5.7)$ & $7(7.4)$ & $0(0)$ & $0(0)$ \\
\hline \multicolumn{5}{|l|}{ Length of work, $n(\%)$} \\
\hline$<1$ year & $4(3.3)$ & $3(3.2)$ & $0(0)$ & $1(5.6)$ \\
\hline $1-5$ years & $5(4.1)$ & $5(5.3)$ & $0(0)$ & $0(0)$ \\
\hline $6-10$ years & $33(26.8)$ & $27(28.4)$ & $5(50.0)$ & $1(5.6)$ \\
\hline$>10$ years & $81(65.9)$ & $60(63.2)$ & $5(50.0)$ & $16(88.9)$ \\
\hline Working at PHCs provides DOT for MDR-TB, $n(\%)$ & $39(31.7)$ & $30(31.6)$ & $4(40.0)$ & $5(27.8)$ \\
\hline Received on-the-job training on DOT for MDR-TB procedure, $n(\%)$ & $36(29.3)$ & $27(28.4)$ & $6(60.0)$ & $3(16.7)$ \\
\hline Received training on infection control, $n(\%)$ & $75(61.0)$ & $61(64.2)$ & $8(80.0)$ & $6(33.3)$ \\
\hline
\end{tabular}

${ }^{a}$ Clinical staff: medical doctor, nurse, and laboratory staff. ${ }^{b}$ Other staff: registration staff, health promotion staff, and environmental health staff PHCs primary health care facilities, DOT direct observed treatment, MDR-TB multidrug-resistant tuberculosis 
Table 2 Distribution of primary health care facilities with optimal infrastructure and activities of infection control

\begin{tabular}{lll}
\hline Domains & Among all observed & Among observed PHCs which provide \\
& PHCs $(N=17)$ & $n$ \\
& $n$ & 1 \\
\hline Optimal infrastructure & & 2 \\
Layout and zoning & 8 & 1 \\
Airflow & 9 & 1 \\
Available exhaust fan & 2 & 0 \\
Maintain exhaust fan & 0 & 7 \\
Available specific infectious waste disposal & 16 & 3 \\
Infectious waste disposal system & 6 & 4 \\
Air ventilation in laboratory & 9 & 5 \\
Specific sputum collection area & 6 & 5 \\
Available hand scrub liquid & 10 & 6 \\
Available and utilized surgical masks for patients & 15 & 4 \\
Optimal activities & & 3 \\
Triage & 14 & 3 \\
Education & 8 & 5 \\
Separation & 10 & 3
\end{tabular}

PHCs primary health care facilities, DOT direct observed treatment, MDR-TB multidrug-resistant tuberculosis

However, their knowledge about other domains of infection control was still suboptimal. Knowledge about timing to provide cough etiquette were higher among clinical and TB program staff compared to other types of staff (registration, environmental health, health promotion staff). The same pattern was found in terms of setting the infrastructure such as a fan for infection control purpose.

Additionally, the quantitative data also showed a low proportion of staff with adequate knowledge of infection

Table 3 Distribution of primary health care staff with adequate knowledge on infection control

\begin{tabular}{|c|c|c|c|c|c|c|c|c|c|c|c|c|c|c|}
\hline \multirow[t]{3}{*}{$\begin{array}{l}\text { Domains of } \\
\text { knowledge }\end{array}$} & \multirow[t]{2}{*}{$\begin{array}{l}\text { Total } \\
(N=123)\end{array}$} & \multicolumn{4}{|l|}{ Type of staff } & \multicolumn{3}{|c|}{$\begin{array}{l}\text { Working at PHCs } \\
\text { which provide } \\
\text { DOT for MDR-TB }\end{array}$} & \multicolumn{3}{|c|}{$\begin{array}{l}\text { Received on-the-job } \\
\text { training on DOT for } \\
\text { MDR-TB procedure }\end{array}$} & \multicolumn{3}{|c|}{$\begin{array}{l}\text { Received training } \\
\text { on infection control }\end{array}$} \\
\hline & & $\begin{array}{l}\text { Clinical staffa } \\
(N=95)\end{array}$ & $\begin{array}{l}\text { TB program } \\
\text { staff }(N=10)\end{array}$ & $\begin{array}{l}\text { Other staff } \\
(N=18)\end{array}$ & $p$ & $\begin{array}{l}\text { Yes } \\
(N=39)\end{array}$ & $\begin{array}{l}\text { No } \\
(N=84)\end{array}$ & $p$ & $\begin{array}{l}\text { Yes } \\
(N=36)\end{array}$ & $\begin{array}{l}\text { No } \\
(N=87)\end{array}$ & $p$ & $\begin{array}{l}\text { Yes } \\
(N=75)\end{array}$ & $\begin{array}{l}\text { No } \\
(N=48)\end{array}$ & $p$ \\
\hline & $n(\%)$ & $n(\%)$ & $n(\%)$ & $n(\%)$ & & $n(\%)$ & $n(\%)$ & & $n(\%)$ & $n(\%)$ & & $n(\%)$ & $n(\%)$ & \\
\hline $\begin{array}{l}\text { Time to provide } \\
\text { education of } \\
\text { cough etiquette } \\
\text { to patients }\end{array}$ & $91(74.0)$ & $72(75.8)$ & $9(90.0)$ & $10(55.6)$ & $0.99^{c}$ & $31(79.5)$ & $60(71.4)$ & 0.343 & 29 (80.6) & $62(71.3)$ & $0.399^{d}$ & $57(76.0)$ & $34(70.8)$ & 0.524 \\
\hline $\begin{array}{l}\text { Cough } \\
\text { screening } \\
\text { among visitors }\end{array}$ & $47(38.2)$ & $41(43.2)$ & $5(50.0)$ & $1(5.6)$ & 0.008 & $11(28.2)$ & $36(42.9)$ & 0.120 & $14(38.9)$ & $33(37.9)$ & 0.921 & $31(41.3)$ & $16(33.3)$ & 0.373 \\
\hline $\begin{array}{l}\text { Proper setting } \\
\text { of fan in } \\
\text { outpatient clinic }\end{array}$ & $88(71.5)$ & $70(73.7)$ & $9(90.0)$ & $9(50)$. & 0.050 & $28(71.8)$ & $60(71.4)$ & 0.967 & $29(80.6)$ & $59(67.8)$ & 0.154 & $59(78.7)$ & $29(60.4)$ & 0.029 \\
\hline $\begin{array}{l}\text { Disposal of } \\
\text { tissues used for } \\
\text { closing mouth } \\
\text { when coughing }\end{array}$ & $105(84.5)$ & 81 (85.3) & $9(90.0)$ & 15 (83.3) & $0.883^{c}$ & $32(82.1)$ & 73 (86.9) & $0.664^{d}$ & 32 (88.9) & $73(83.9)$ & $0.667^{d}$ & $64(85.3)$ & $41(85.4)$ & 1.00 \\
\hline $\begin{array}{l}\text { Use of } \\
\text { respiratory mask }\end{array}$ & $60(48.8)$ & $48(50.5)$ & $5(50.0)$ & $7(38.9)$ & 0.661 & $20(51.3)$ & $40(47.6)$ & 0.705 & $18(50.0)$ & $42(48.3)$ & 0.862 & $37(49.3)$ & $23(47.9)$ & 0.878 \\
\hline $\begin{array}{l}\text { Use surgical } \\
\text { masks among } \\
\text { patients during } \\
\text { transportation }\end{array}$ & $81(65.9)$ & $58(61.1)$ & $7(70.0)$ & $16(88.9)$ & 0.071 & $30(76.9)$ & $51(60.7)$ & 0.078 & $28(77.8)$ & $53(60.9)$ & 0.073 & $50(66.7)$ & $31(64.6)$ & 0.812 \\
\hline
\end{tabular}

${ }^{a}$ Clinical staff: medical doctor, nurse, and laboratory staff. ${ }^{b}$ Other staff: registration staff, health promotion staff, and environmental health staff. In general, the data analysis used the Pearson chi-square test. Few analyses used the likelihood ratio chi-square test ${ }^{c}$ and chi-square test with continuity correction ${ }^{d}$

$T B$ tuberculosis, PHCs primary health care facilities, DOT direct observed treatment, MDR-TB multidrug-resistant tuberculosis 
control in two domains: cough screening among visitors $(38 \%)$ and use of a respiratory mask (48\%) (Table 3). Knowledge about cough screening was lower among staff who were not directly involved in the process of DOT for MDR-TB patients (e.g., registration staff, health promotion, or environmental health staff) compared to clinical or TB program staff ( $p$ value $=0.008$ ). There was no statistically significant difference between staff working at PHCs with DOT for MDR-TB service and those working in PHCs without DOT for MDR-TB service in terms of knowledge of staff about cough $(p$ value $=$ $0.120)$ and the use of a respiratory mask ( $p$ value $=$ 0.705). We also found a similar pattern when we compared between staff who received training on DOT for MDR-TB and infection control with those who did not.

\section{Feeling safe}

Our quantitative data showed that approximately half of the health staff stated about feeling stressed and fearful in conducting MDR-TB care (Table 4). Staff at a PHC providing DOT for MDR-TB service perceived less burden of workload compared to the ones at a PHC without the DOT service $(56 \%$ vs. $32 \%, p$ value $=0.011)$. However, there was a relatively similar proportion of staff with perceived fear to be infected or feeling afraid to talk with MDR-TB patients by the type of staff, working/not working at PHCs with DOT, and history of trainings on DOT or infection control.
The findings from the cross-sectional survey also confirmed a lack of confidence and perceived capacity to provide MDR-TB care in the PHCs (Table 5). Only 65\% of health staff felt confident in conducting DOT for MDR-TB patients. Less than $60 \%$ of health staff reported their understanding of DOT procedures. Similarly, only $53 \%$ of total staff stated that infection control is properly managed in the PHCs. Clinical and TB program staff showed lower confidence in the infection control in PHCs compared to other staff ( $p$ value $=0.020$ ).

Our study also showed the lack of staff motivation to conduct MDR-TB care. Only 67\% showed their commitment to providing DOT in accordance to proper protocols for MDR-TB patients. Notably, 26\% perceived their preference not to provide proper care, when it was considered a choice (Table 5).

The observation showed inefficiency in the use of the infection control infrastructure of infection control in our observations. For illustration, in a DOT room outside the PHC building that had much airflow, there was an ordinary fan and exhaust fan had been set up. Both fans were used when DOT was provided.

The feeling of being unsafe was also reflected by unnecessary practices by health staff such as wearing double hand gloves, waterproofed gown, plastic boots, and double masks. Those practices reflected efforts to have maximum protection. As an illustration, a nurse at the PHC stated, "I am afraid of being infected... [I know that] the PHC staff who conducts the DOT wear

Table 4 Distribution of primary health care staff who stated perceived stress and fear to conduct multidrug-resistant tuberculosis care

\begin{tabular}{|c|c|c|c|c|c|c|c|c|c|c|c|c|c|c|}
\hline \multirow[t]{3}{*}{$\begin{array}{l}\text { Domains } \\
\text { of attitude }\end{array}$} & \multirow[t]{2}{*}{$\begin{array}{l}\text { Total } \\
(N=123)\end{array}$} & \multicolumn{4}{|l|}{ Type of staff } & \multicolumn{3}{|c|}{$\begin{array}{l}\text { Working at PHCs } \\
\text { which provide } \\
\text { DOT for MDR-TB }\end{array}$} & \multicolumn{3}{|c|}{$\begin{array}{l}\text { Received on-the-job } \\
\text { training on DOT for } \\
\text { MDR-TB procedure }\end{array}$} & \multicolumn{3}{|c|}{$\begin{array}{l}\text { Received training } \\
\text { on infection control }\end{array}$} \\
\hline & & $\begin{array}{l}\text { Clinical staffa } \\
(N=95)\end{array}$ & $\begin{array}{l}\text { TB program } \\
\text { staff }(N=10)\end{array}$ & $\begin{array}{l}\text { Other staffo } \\
(N=18)\end{array}$ & $p$ & $\begin{array}{l}\text { Yes } \\
(N=39)\end{array}$ & $\begin{array}{l}\text { No } \\
(N=84)\end{array}$ & $p$ & $\begin{array}{l}\text { Yes } \\
(N=36)\end{array}$ & $\begin{array}{l}\text { No } \\
(N=87)\end{array}$ & $p$ & $\begin{array}{l}\text { Yes } \\
(N=75)\end{array}$ & $\begin{array}{l}\text { No } \\
(N=48)\end{array}$ & $p$ \\
\hline & $n(\%)$ & $n(\%)$ & $n(\%)$ & $n(\%)$ & & $n(\%)$ & $n(\%)$ & & $n(\%)$ & $n(\%)$ & & $n(\%)$ & $n(\%)$ & \\
\hline \multicolumn{15}{|l|}{ Stress } \\
\hline $\begin{array}{l}\text { Taking account } \\
\text { of my workload, } \\
\text { I am still able to } \\
\text { provide DOT for } \\
\text { MDR-TB patients. }\end{array}$ & $\begin{array}{l}49 \\
(39.9)\end{array}$ & $\begin{array}{l}36 \\
(37.9)\end{array}$ & $\begin{array}{l}5 \\
(50.0)\end{array}$ & $\begin{array}{l}8 \\
(44.4)\end{array}$ & 0.691 & $\begin{array}{l}22 \\
(56.4)\end{array}$ & $\begin{array}{l}27 \\
(32.1)\end{array}$ & 0.011 & $\begin{array}{l}21 \\
(58.3)\end{array}$ & $\begin{array}{l}28 \\
(32.2)\end{array}$ & 0.007 & $\begin{array}{l}32 \\
(42.7)\end{array}$ & $\begin{array}{l}17 \\
(35.4)\end{array}$ & 0.423 \\
\hline $\begin{array}{l}\text { I feel stressed } \\
\text { providing } \\
\text { DOT for } \\
\text { MDR-TB patients. }\end{array}$ & $\begin{array}{l}65 \\
(52.8)\end{array}$ & $\begin{array}{l}14 \\
(14.7)\end{array}$ & $\begin{array}{l}2 \\
(20.0)\end{array}$ & $\begin{array}{l}5 \\
(27.8)\end{array}$ & 0.425 & $\begin{array}{l}4 \\
(10.3)\end{array}$ & $\begin{array}{l}17 \\
(20.2)\end{array}$ & 0.171 & $\begin{array}{l}2 \\
(5.6)\end{array}$ & $\begin{array}{l}19 \\
(21.8)\end{array}$ & $0.055^{c}$ & $\begin{array}{l}15 \\
(20.0)\end{array}$ & $\begin{array}{l}6 \\
(12.5)\end{array}$ & $0.405^{c}$ \\
\hline \multicolumn{15}{|l|}{ Fear } \\
\hline $\begin{array}{l}\text { I am afraid to be } \\
\text { infected when } \\
\text { I provide DOT for } \\
\text { MDR-TB patients. }\end{array}$ & $\begin{array}{l}58 \\
(47.1)\end{array}$ & $\begin{array}{l}44 \\
(46.3)\end{array}$ & $\begin{array}{l}5 \\
(50.0)\end{array}$ & $\begin{array}{l}9 \\
(50.0)\end{array}$ & 0.943 & $\begin{array}{l}18 \\
(46.2)\end{array}$ & $\begin{array}{l}40 \\
(47.6)\end{array}$ & 0.880 & $\begin{array}{l}13 \\
(36.1)\end{array}$ & $\begin{array}{l}45 \\
(51.7)\end{array}$ & 0.115 & $\begin{array}{l}35 \\
(46.7)\end{array}$ & $\begin{array}{l}23 \\
(47.9)\end{array}$ & 0.892 \\
\hline $\begin{array}{l}\text { I am not afraid to } \\
\text { talk with MDR-TB } \\
\text { patients when I } \\
\text { provide DOT. }\end{array}$ & $\begin{array}{l}58 \\
(47.2)\end{array}$ & $\begin{array}{l}43 \\
(45.3)\end{array}$ & $\begin{array}{l}5 \\
(50.0)\end{array}$ & $\begin{array}{l}10 \\
(55.6)\end{array}$ & 0.712 & $\begin{array}{l}19 \\
(48.7)\end{array}$ & $\begin{array}{l}39 \\
(46.4)\end{array}$ & 0.813 & $\begin{array}{l}17 \\
(47.2)\end{array}$ & $\begin{array}{l}41 \\
(47.1)\end{array}$ & 0.992 & $\begin{array}{l}39 \\
(52.0)\end{array}$ & $\begin{array}{l}19 \\
(39.6)\end{array}$ & 0.178 \\
\hline
\end{tabular}

${ }^{a}$ Clinical staff: medical doctor, nurse, and laboratory staff. ${ }^{b}$ Other staff: registration staff, health promotion staff, and environmental health staff. In general, the data analysis used the Pearson chi-square test. Few analyses used the chi-square test with continuity correction ${ }^{c}$

TB tuberculosis, PHCs primary health care facilities, DOT direct observed treatment, MDR-TB multidrug-resistant tuberculosis 
Table 5 Distribution of primary health care staff who agree about perceived capacity and motivation to conduct direct observed treatment for multidrug-resistant tuberculosis patients

\begin{tabular}{|c|c|c|c|c|c|c|c|c|c|c|c|c|c|c|}
\hline \multirow[t]{3}{*}{ Domains of attitude } & \multirow[t]{2}{*}{$\begin{array}{l}\text { Total } \\
(N=123)\end{array}$} & \multicolumn{4}{|l|}{ Type of staff } & \multicolumn{3}{|c|}{$\begin{array}{l}\text { Working at PHCs } \\
\text { which provide } \\
\text { DOT for MDR-TB }\end{array}$} & \multicolumn{3}{|c|}{$\begin{array}{l}\text { Received on-the- } \\
\text { job training on } \\
\text { DOT for MDR-TB } \\
\text { procedure }\end{array}$} & \multicolumn{3}{|c|}{$\begin{array}{l}\text { Received training } \\
\text { on infection } \\
\text { control }\end{array}$} \\
\hline & & $\begin{array}{l}\text { Clinical staffa } \\
(N=95)\end{array}$ & $\begin{array}{l}\text { TB program } \\
\text { staff }(N=10)\end{array}$ & $\begin{array}{l}\text { Other staff } \\
(N=18)\end{array}$ & $p$ & $\begin{array}{l}\text { Yes } \\
(N=39)\end{array}$ & $\begin{array}{l}\text { No } \\
(N=84)\end{array}$ & $p$ & $\begin{array}{l}\text { Yes } \\
(N=36)\end{array}$ & $\begin{array}{l}\text { No } \\
(N=87)\end{array}$ & $p$ & $\begin{array}{l}\text { Yes } \\
(N=75)\end{array}$ & $\begin{array}{l}\text { No } \\
(N=48)\end{array}$ & $p$ \\
\hline & $n(\%)$ & $n(\%)$ & $n(\%)$ & $n(\%)$ & & $n(\%)$ & $n(\%)$ & & $n(\%)$ & $n(\%)$ & & $n(\%)$ & $n(\%)$ & \\
\hline \multicolumn{15}{|l|}{ Perceived capacity } \\
\hline $\begin{array}{l}\text { I am confident } \\
\text { conducting DOT } \\
\text { for MDR-TB patient. }\end{array}$ & $\begin{array}{l}81 \\
(65.9)\end{array}$ & $\begin{array}{l}62 \\
(65.3)\end{array}$ & $\begin{array}{l}9 \\
(90.0)\end{array}$ & $\begin{array}{l}10 \\
(55.6)\end{array}$ & 0.178 & $\begin{array}{l}32 \\
(82.1)\end{array}$ & $\begin{array}{l}49 \\
(58.3)\end{array}$ & 0.01 & $\begin{array}{l}31 \\
(86.1)\end{array}$ & $\begin{array}{l}50 \\
(57.5)\end{array}$ & 0.002 & $\begin{array}{l}52 \\
(69.3)\end{array}$ & $\begin{array}{l}29 \\
(60.4)\end{array}$ & 0.309 \\
\hline $\begin{array}{l}\text { I understand the } \\
\text { DOT procedure } \\
\text { for MDR-TB patient. }\end{array}$ & $\begin{array}{l}72 \\
(58.6)\end{array}$ & $\begin{array}{l}58 \\
(61.1)\end{array}$ & $\begin{array}{l}7 \\
(70.0)\end{array}$ & $\begin{array}{l}7 \\
(38.9)\end{array}$ & 0.161 & $\begin{array}{l}31 \\
(79.5)\end{array}$ & $\begin{array}{l}41 \\
(48.8)\end{array}$ & 0.001 & $\begin{array}{l}32 \\
(88.9)\end{array}$ & $\begin{array}{l}40 \\
(46.0)\end{array}$ & $<0.001$ & $\begin{array}{l}48 \\
(64.0)\end{array}$ & $\begin{array}{l}24 \\
(50.0)\end{array}$ & 0.124 \\
\hline $\begin{array}{l}\text { Infection control is } \\
\text { properly managed } \\
\text { in the } \mathrm{PHC} \text {. }\end{array}$ & $\begin{array}{l}66 \\
(53.7)\end{array}$ & $\begin{array}{l}47 \\
(49.5)\end{array}$ & $\begin{array}{l}4 \\
(40.0)\end{array}$ & $\begin{array}{l}15 \\
(83.3)\end{array}$ & 0.020 & $\begin{array}{l}24 \\
(61.5)\end{array}$ & $\begin{array}{l}42 \\
(50.0)\end{array}$ & 0.232 & $\begin{array}{l}20 \\
(55.6)\end{array}$ & $\begin{array}{l}46 \\
(52.9)\end{array}$ & 0.786 & $\begin{array}{l}36 \\
(48.0)\end{array}$ & $\begin{array}{l}30 \\
(62.5)\end{array}$ & 0.116 \\
\hline \multicolumn{15}{|l|}{ Motivation } \\
\hline $\begin{array}{l}\text { If there were a choice, } \\
\text { I would prefer not to } \\
\text { conduct DOT for } \\
\text { MDR-TB patients. }\end{array}$ & $\begin{array}{l}33 \\
(26.8)\end{array}$ & $\begin{array}{l}28 \\
(29.5)\end{array}$ & $\begin{array}{l}1 \\
(10.0)\end{array}$ & $\begin{array}{l}9 \\
(22.2)\end{array}$ & 0.319 & $\begin{array}{l}9 \\
(23.1)\end{array}$ & $\begin{array}{l}24 \\
(28.6)\end{array}$ & 0.522 & $\begin{array}{l}6 \\
(16.7)\end{array}$ & $\begin{array}{l}27 \\
(31.0)\end{array}$ & 0.158 & $\begin{array}{l}17 \\
(22.7)\end{array}$ & $\begin{array}{l}16 \\
(33.3)\end{array}$ & 0.193 \\
\hline $\begin{array}{l}\text { I think I should be } \\
\text { involved in TB control } \\
\text { by doing DOT for } \\
\text { MDR-TB patients. }\end{array}$ & $\begin{array}{l}83 \\
(67.5)\end{array}$ & $\begin{array}{l}60 \\
(63.2)\end{array}$ & $\begin{array}{l}10 \\
(100)\end{array}$ & $\begin{array}{l}13 \\
(72.2)\end{array}$ & 0.055 & $\begin{array}{l}28 \\
(71.8)\end{array}$ & $\begin{array}{l}55 \\
(65.5)\end{array}$ & 0.486 & $\begin{array}{l}30 \\
(83.3)\end{array}$ & $\begin{array}{l}53 \\
(60.9)\end{array}$ & 0.016 & $\begin{array}{l}57 \\
(76.0)\end{array}$ & $\begin{array}{l}26 \\
(54.2)\end{array}$ & 0.012 \\
\hline
\end{tabular}

${ }^{a}$ Clinical staff: medical doctor, nurse, and laboratory staff. ${ }^{\mathrm{b}}$ Other staff: registration staff, health promotion staff, and environmental health staff. Data analysis used the Pearson chi-square test

$T B$ tuberculosis, $P H C$ s primary health care facilities, $D O T$ direct observed treatment, MDR-TB multidrug-resistant tuberculosis

excessive personal protective equipment... I am laughing at them, but I am also afraid... An accident can happen. If we get infected, what is the guarantee? Let's see, some of our friends got infected, what do they get? Even we are blamed, because of the lack of personal protective equipment..." (Health staff 11, a nurse).

Feeling safe or unsafe to conduct DOT for MDR-TB patients was an interaction between four domains: fear, perceived risk, security, and adequate training (Table 6). Fear was perceived as a health staff's natural expression when taking care of patients with MDR-TB. However, the perceived risk of being infected varied:

We are at high risk of getting infected, however, there is no certainty on our insurance if we are infected (Health staff 9, a nurse)

Masks have been provided at the registration desk. There were TB patients here, but health staffs did not wear it. Moreover, there will be an MDR TB patient here (Health staff 13, a nurse who was also a TB program staff)

The awareness about the risk of being infected increased when the PHC received an MDR-TB patient for DOT, as expressed by an informant, "They (health staffs) were shocked when they found out there was an MDR-TB patient here" (Health staff 16, a nurse who was also a TB program staff).

Perceived risk and lacking security among the staff prompted the domination of fear in their response in regard to DOT for MDR-TB patients. While training was considered as an intervention to reduce the fear, infection control capacity in the PHC was essential. Training only was perceived could not overcome fear.

...Our intention is to help patients, and we have no choice. We are sure we will be safe as long as our infection control is adequate. (Health staff 11, a nurse)

We provided training to our staff, but it could not overcome fear. (Health staff 6 , a physician)

Information from the in-depth interviews showed that the coverage and quality of training were perceived inadequate by some health staff at PHCs. The survey data showed that only $29 \%$ of health staff received the training on DOT for the MDR-TB procedure. Our confirmation with the TB program managers revealed that the training was conducted in a working day at the PHC as a preparation to receive a back referred patient from the MDR-TB center hospital. 
Table 6 Examples of meaning units, sub-themes and themes from content analysis of in-depth interviews data about the perception of staff in relation to Directly Observed Treatment for Multidrug Resistant Tuberculosis

\begin{tabular}{|c|c|c|c|c|}
\hline Meaning unit & $\begin{array}{l}\text { Condensed meaning unit } \\
\text { Description close to the text }\end{array}$ & $\begin{array}{l}\text { Condensed meaning unit } \\
\text { Interpretation of the } \\
\text { underlying meaning }\end{array}$ & Sub-theme & Theme \\
\hline $\begin{array}{l}\text { "Our main challenge is health staff's fear } \\
\text { toward MDR-TB. One of the factors is } \\
\text { uneven knowledge distribution among } \\
\text { health staffs. The more we know }\end{array}$ & $\begin{array}{l}\text { Health staff's fear toward } \\
\text { MDR-TB }\end{array}$ & $\begin{array}{l}\text { Fear is a natural } \\
\text { expression of health } \\
\text { staff when they } \\
\text { know about MDR-TB }\end{array}$ & Fear & $\begin{array}{l}\text { Feeling safe/unsafe as an } \\
\text { interaction between fear, } \\
\text { perceived risk and security, } \\
\text { as well as level of knowledg }\end{array}$ \\
\hline
\end{tabular}

(about MDR-TB), the more we are scared" (Health staff 4)

"Health staffs were scared to get infected. Fear is human, is not it?" (Health staff 1)

"They (health staffs) were shocked when they found out there was an MDR-TB patient here" (Health staff 16)

"Masks have been provided at the registration desk. There were TB patients here, but health staffs did not wear it. Moreover, there will be MDR-TB patient here" (Health staff 13)

"We are at high risk of getting infected, however there is no certainty on our insurance if we are infected" (health staff 9)

"They are scared to death. There is no insurance or guarantee of safety from the management" (Health staff 5)

"... Our intention is to help patient, and we have no choice. We are sure we will be safe as long as our infection control is adequate" (Health staff 11)

"We will not get infected if the Infection Control is adequate" (Health staff 11)

"First of all, they were afraid to get infected and they are questioning whether the Infection Control has been adequate yet" (Health staff 10)

"It will be useless to give training to the health staff. If they are scared, it's personal..." (Health staff 2)

"When they (health staffs) were surveyed, the result might show low knowledge.

It is because uneven knowledge distribution among health staffs" (Health staff 1)

"... Because we have not got the training so our fear beats everything" (Health staff 10)

"Most of health staffs only focus on their job, for instance nurse and nutritionist did not understand about this matter (MDR-TB)" (Health staff 1)

"There is indeed fear toward MDR-TB. Health staffs should understand on infection control of MDR-TB. Therefore, dissemination of information is very important"
Fear is human

Fear is a natural expression

Health staff were scared

to get infected

The health staff were shocked when they found out there was an MDR-TB patient at the $\mathrm{PHC}$

The health staff (at the Awareness about risk of registration desk) did not wear being infected is low mask, while there will be MDR

$\mathrm{TB}$ patient (at the $\mathrm{PHC}$ )

No certainty on our insurance if we are infected

Lack security for staff in doing DOT for MDR-TB patients.

There is no insurance or guarantee of safety from the management

We will be safe as long as our infection control is adequate

The health staff will not get infected if the infection control is adequate

Health staff are questioning whether the infection control has been adequate yet

It will be useless to give training to give training

Uneven knowledge distribution among

health staff

Health staff have not got the training

The health staff did not understand about MDR-TB

Dissemination of information (about infection control of MDR-TB) is important
The infection control is perceived inadequate

Infection control application is perceived essential to prevent MDR-TB transmission

The infection control is perceived inadequate

Current content of training can be not effective to reduce fear

Need more staff to be trained

Fear is bigger when the staff have not received training

Information about MDR-TB is still limited among health staff
Perceived risk

Perceived security well as level of knowledge. 
Table 6 Examples of meaning units, sub-themes and themes from content analysis of in-depth interviews data about the perception of staff in relation to Directly Observed Treatment for Multidrug Resistant Tuberculosis (Continued)

\begin{tabular}{|c|c|c|c|c|}
\hline Meaning unit & $\begin{array}{l}\text { Condensed meaning unit } \\
\text { Description close to the text }\end{array}$ & $\begin{array}{l}\text { Condensed meaning unit } \\
\text { Interpretation of the } \\
\text { underlying meaning }\end{array}$ & Sub-theme & Theme \\
\hline $\begin{array}{l}\text { "Overall, PHCs are ready. There are only } \\
\text { few health staff who still discriminate } \\
\text { MDR-TB patient because of scared" } \\
\text { (Health staff 2) }\end{array}$ & $\begin{array}{l}\text { Few health staff discriminate } \\
\text { MDR-TB patients because of } \\
\text { scared. }\end{array}$ & $\begin{array}{l}\text { Fear is a driver to a } \\
\text { stigmatic attitude to } \\
\text { MDR-TB patients }\end{array}$ & $\begin{array}{l}\text { Stigmatic attitude } \\
\text { Fear }\end{array}$ & $\begin{array}{l}\text { Fear as a driver of } \\
\text { stigmatic attitude }\end{array}$ \\
\hline
\end{tabular}

\section{Stigmatic attitude}

The stigmatic attitude was also shown in our observation. Some health staff kept unnecessary distances from the patients when they conducted DOT for MDR-TB care. Even a health staff was talking with the patient from a small window while the patient was in the DOT room and also locked the door of the DOT room. The in-depth interview data revealed that fear was a driver to a stigmatic attitude to MDR-TB patients.

\section{Discussions}

Our study highlighted existing issues about being safe, feeling safe, and stigmatic attitudes among health staff in providing MDR-TB care. Some infrastructures of infection control in PHCs are suboptimal. There was also lacking knowledge of infection control protocols. The stigmatic attitudes manifest in various forms: fear of being infected, avoidance to conduct care, and performing unnecessary overprotected practices of infection control. The cause of the stigmatizing attitude was the fear of being infected. The combination of the suboptimal infection control environment, lacking knowledge, and stigma resulted in unsafe and inefficient practice while doing the MDR-TB care, as well as the discrimination of MDR-TB patients.

Our findings on the inadequacy knowledge and practices of TB infection control such as patients' screening corroborate previous findings by studies in the hospital setting in South Africa $[17,18]$. Low level of knowledge was also worse among administrative staff and other staff who do not have direct relevance to MDR-TB care [19]. Other previous studies in India also found problems with the infection control infrastructure related to drug-resistant TB $[20,21]$.

The fear among health staff due to MDR-TB transmission risk, while they provide care, is the cause of stigmatizing attitude, as was also found in the previous research studies [22-24]. Stigma among health staff is hypothesized to influence the practice of infection control [23]. Our data revealed empirical evidence that the fear even induced the unsafe and inefficient method of infection control by wearing binary masks (respiratory and surgical masks) and hand gloves. Our study also provided empirical evidence about the influence of fear in the communication between provider and patient that was also proposed by others [22].

\section{Public health implications}

The state of being safe is a priority issue that should be addressed by improving the quality of infection control. Our finding showed a need for knowledge and capacity building for infection control. We argue that this intervention will be a foundation to influence the feeling of safety among health workers to conduct MDR-TB care.

Our study added the gap of knowledge about whether the stigma of health staff to conduct TB care is/is not reasonable. The internalized and enacted stigma among health staff is found related to feeling unsafe due to a lacking infection control infrastructure and knowledge. Improved supervision of MDR-TB care should be performed with the continuation of its implementation.

The findings of stigmatic stigma signified the need for improvement of the quality of care provided by health staff at PHCs. Considering that patient-centered care is one of the pillars of the strategy to end TB [1], we agreed with others that the improvement of the quality of MDR-TB care should include empathy and respect for the patients [24].

Interventions to reduce stigma concerning TB among health care providers are limited [25]. Wu et al.'s study put an effort to decrease internalized stigma among health care workers by training to improve knowledge and attitude among health staff; however, it has been shown less effective among health staff conducting DOT [26]. Hence, more research is needed to get pieces of evidence about the effective strategies to reduce stigma among health staff providing MDR-TB care.

\section{Study limitation and strength}

Our study aimed to explore the phenomenon related to the rejection of the staff to conduct MDR-TB direct observed treatment cases. The study design could not assess any causal relationship between issues of safety, feeling safe, and stigmatizing attitude. On the other hand, the findings from the qualitative data could enrich the interpretation of the quantitative data. Therefore, we could have a holistic portrait of the phenomenon.

This study explored the phenomena of safety and stigmatic attitude with qualitative study which was limited in the generalization in other settings. In future, stigma should be measured quantitatively using a locally adapted instrument. The measurement will be useful to 
support the evidence related to the level of stigma. Since we argue with the urged need to reduce stigma, the quantitative measurement of stigma among health staff will support the evaluation of the effect from the stigma reduction initiative.

Our study added the gap of knowledge about whether the stigma of health staff to conduct TB care is/is not reasonable in the Indonesian context. We found that the internalized and enacted stigma among health staff is found related to feeling unsafe due to a lacking infection control infrastructure and knowledge. Improved supervision of MDR-TB care should be performed with the continuation of its implementation. The findings from our study contributed to current efforts to understand the nature of stigma among health staff in conducting MDR-TB care that is argued as an initial step to address and tacking the implementation issue of DOT for MDR-TB patients [27]. The way forward to patient-centered MDR-TB care would be jeopardized unless stigma among health staff is diminished.

\section{Conclusion}

Our study concluded with a clear demonstration of lacking safety, feeling unsafe, and stigmatic attitude among health staff to conduct patient-centered MDR-TB care in $\mathrm{PHCs}$ in Indonesia. Serious efforts are needed on all levels to ensure safety and prevent irrational stigma.

\section{Abbreviations \\ DOT: Direct observed treatment; MDR-TB: Multidrug-resistant tuberculosis; PHC: Primary health care facility; TB: Tuberculosis}

\section{Acknowledgements}

We give high appreciation to all study participants. We appreciate Prof Daniel D. Reidpath, Prof. Pascale Allotey, Dr. Yodi Mahendradhata, Dr. Siwi Retna Patmawati, Dr. Vitri Widyaningsih and Dr. Riris Andono Ahmad for their inputs to this manuscript.

\section{Funding}

This research received financing for research project and publication from The Center for Health Policy and Management, Faculty of Medicine, Universitas Gadjah Mada in collaboration with the Alliance for Health Policy \& Systems Research (AHPSR), the Special Programme for Research, Development and Research Training in Human Reproduction (HRP), and the Special Program for Research and Training on Tropical Diseases (TDR)

\section{Availability of data and materials}

The datasets generated and/or analyzed during the current study are not publicly available due there was not a part of the informed consent. However, the data available from the corresponding author on reasonable request.

\section{Authors' contributions}

AP developed the study, analyzed and interpreted both quantitative and qualitative data, and was a major contributor in writing the manuscript. HS developed the study and collected and analyzed the qualitative data. MRM collected and analyzed the quantitative data. LA analyzed the qualitative data. SW developed the study and collected and analyzed the quantitative data. All authors read and approved the final manuscript.

\section{Ethics approval and consent to participate}

The Ethics Committee Faculty of Medicine Universitas Sebelas Maret, Surakarta, Indonesia, approved this research. The researchers performed written informed consent from all study participants.
Consent for publication

Not applicable.

\section{Competing interests}

The authors declare that they have no competing interests.

\section{Publisher's Note}

Springer Nature remains neutral with regard to jurisdictional claims in published maps and institutional affiliations.

\section{Author details}

'Department of Public Health, Faculty of Medicine, Universitas Sebelas Maret, Jalan Ir. Sutami 36A, Surakarta 57126, Indonesia. ${ }^{2}$ Disease Control Research Group, Faculty of Medicine, Universitas Sebelas Maret, Jalan Ir. Sutami 36A, Surakarta 57126, Indonesia. ${ }^{3}$ Bantul District Health Office, Komplek II Kantor Pemerintah Kabupaten Bantul Jalan Lingkar Timur, Manding, Trirenggo, Bantul, Yogyakarta Province 55714, Indonesia.

Received: 1 February 2018 Accepted: 15 February 2019

Published online: 04 March 2019

\section{References}

1. WHO. The End TB Strategy. https://www.who.int/tb/strategy/end-tb/en/ Accessed 18 December 2017.

2. United Nations. The 2014 UNGA resolution on health worker protection. http://www.who.int/workforcealliance/media/news/2014/UN_Dec2014 health_worker_protection_res.pdf?ua=1. Accessed 18 December 2017.

3. Dodor EA, Kelly SJ. Manifestations of tuberculosis stigma within the healthcare system: the case of Sekondi-Takoradi metropolitan district in Ghana. Health Policy. 2010;98(2-3):195-202.

4. Skinner D, Classens M. It's complicated: why do tuberculosis patients not initiate or stay adherent to treatment? A qualitative study from South Africa. BMC Infect Dis. 2016;16:712.

5. Ahmedani BK. Mental health stigma: society, individuals, and the profession. J Soc Work Values Ethics. 2011;8(2):4 -1-4-16.

6. WHO. Global Tuberculosis Report. $20^{\text {th }}$ edition. Geneva: WHO; 2015.

7. Deacon H. Towards a sustainable theory of health-related stigma: lessons from the HIV/AIDS literature. J Community Appl Soc Psychol. 2006;16:418-25.

8. WHO South-East Asia. What is DOTS (Directly Observed Treatment, Short Course) http://www.searo.who.int/tb/topics/what_dots/en/. Accessed 18 Dec 2017.

9. Frieden TR, Sbarbaro JA. Promoting adherence to treatment for tuberculosis: the importance of direct observation. Bull World Health Organ. 2007:85(5):407-9.

10. District Health Office of Bantul. District Health Profile 2016 (Profil Kesehatan 2017). https://dinkes.bantulkab.go.id/filestorage/dokumen/2017/10/ narasi\%20profil\%202017.pdf. Acessed 18 December 2017.

11. Creswell JW, Plano Clark VL. Designing and conducting mixed methods research. Los Angeles: Sage publication; 2007.

12. Dean AG, Sullivan KM, Soe MM. OpenEpi: Open Source Epidemiologic Statistics for Public Health, Version. www.OpenEpi.com, updated 2013/04/ 06, accessed 2018/10/15.

13. Ministry of Health Republic of Indonesia. Pedoman Pencegahan dan Pengendalian Infeksi Tuberkulosis di Fasilitas Pelayanan Kesehatan. Kementerian Kesehatan RI Direktorat Bina Upaya Kesehatan Jakarta, 2012.

14. Graneheim UH, Lundman B. Qualitative content analysis in nursing research: concepts, procedures and measures to achieve trustworthiness. Nurse Educ Today. 2004;24:105-12.

15. Hsieh HF, Shannon SE. Three approaches to qualitative content analysis. Qual Health Res. 2005;15:1277-87.

16. CT Services and System Development and Division of Epidemiology and Global Health Umeå University. OpenCode 3.4. https://www.umu.se/en/ department-of-epidemiology-and-global-health/research/open-code2/. Accessed 27 September 2017.

17. Kanjee Z, Catterick K, Moll AP, Amico KR, Friedland GH. Tuberculosis infection control in rural South Africa: survey of knowledge, attitude, and practice in hospital staff. J Hosp Infect. 2011;79:333-8.

18. Flick RJ, Munthali A, Simon K, Hosseinipour M, Kim MH, Mlauzi L, et al. Assesing infection control practices to protect health care workers and patients in Malawi from nosocomial transmission of Mycobacterium tuberculosis. PloS One. 2017;12(12):e0189140. 
19. Shrestha A, Bhattarai D, Thapa B, Basel P, Wagle RR. Health care workers' knowledge, attitudes and practices on tuberculosis infection control, Nepal. BMC Infect Dis. 2017;17:724.

20. Akshaya KM, Shewade HD, Aslesh OP, Nagaraja SB, Nirgude AS

Singarajipura A, et al. "Who has to do it at the end of the day? Programme officials or hospital authorities?" Airborne infection control at drug resistant tuberculosis (DR-TB) centres of Karnataka, India: a mixed-methods study. Antimicrob Resist Infect Control. 2017;6:111.

21. Waheed Y, Khan MA, Fatima R, Yagoob A, Mirza A, et al. Infection control in hospitals managing drug-resistant tuberculosis in Pakistan: how are we doing? Public Health Action. 2017;7(1):26-31.

22. Courtwright A, Turner AN. Tuberculosis and stigmatization: pathways and interventions. Public Health Rep. 2010;Supp 4(125):34-42.

23. Macintyre K, Bakker MI, Bergson S, Bhavaraju R, Bond V, Chikovore J, et al. Defining the research agenda to measure and reduce tuberculosis stigmas. Int J Tuberc Lung Dis. 2017;21(11):S87-96.

24. Tadesse S. Stigma against tuberculosis patients in Addis Ababa, Ethiopia. PLoS One. 2016;11(4):e0152900. https://doi.org/10.1371/journal.pone.0152900.

25. Sommerland N, Wouters W, Mitchell EMH, Ngicho M, Redwood L, Masquillier C, et al. Evidence-based interventions to reduce tuberculosis stigma: a systematic review. Int J Tuberc Lung Dis. 2017;21(11):S81-6.

26. Wu PS, Chou P, Chang NT, Sun WJ, Kuo HS. Assessment of changes in knowledge and stigmatization following tuberculosis training workshops in Taiwan. J Formos Med Assoc. 2009;108(5):377-85.

27. Jaramillo E, Sahu S, Van Weezenbeek C. Ending TB-related stigma and discrimination. Int J Tuberc Lung Dis. 2017;21(11):S2-3.

Ready to submit your research? Choose BMC and benefit from:

- fast, convenient online submission

- thorough peer review by experienced researchers in your field

- rapid publication on acceptance

- support for research data, including large and complex data types

- gold Open Access which fosters wider collaboration and increased citations

- maximum visibility for your research: over $100 \mathrm{M}$ website views per year

At $\mathrm{BMC}$, research is always in progress.

Learn more biomedcentral.com/submissions 